\title{
A Kinematics Analysis of Back Gliding Shot Put Techniques of Xiangrong Liu
}

\author{
Weiwei Zhang, Jihe Zhou* and Jianhong Xu \\ Chengdu Sport Institute, Chengdu China 610041 \\ ${ }^{*}$ Corresponding author
}

\begin{abstract}
Taking Xiangrong Liu, a Chinese female shot athlete, as the research object, the biomechanical analysis of the technical action of its backward slide-shot putting using the method of sports biomechanics was carried out. The study found that: in the gliding stage, Xiangrong Liu's maximum speed of velocity of the shot and the maximum center of gravity of the body weight difference, the trunk lift amplitude is too large; support time in the transitional phase is too long; swing leg is not actively inserted; in the last force phase, she shot slower and the angle of shot is too large.
\end{abstract}

\section{Keywords—athletes; back-gliding; shot; kinematics}

\section{RESEARCH PURPOSES}

There have been a number of elite athletes from Chinese Female Shot Put Team in the World Track and Field, and the women putters usually obtained gratifying results in the world track and field competition. However, those years, the level and grades of Chinese Female Shot Put are showing a downward trend, which is lower and lower than the level of world female shot put. This dissertation provides scientific theoretical bases for modifying the mistaken movements in the process of backward gliding shot put, through the deconstruction of Xiangrong Liu's backward slide shot put 3D video, obtaining relative kinematic statistics. (Xiangrong Liu, the champion of the female shot put of China Throwing GameChengdu Site.)

\section{RESEARCH OBJECTS AND METHODS}

\section{A. Research Objects}

Xiangrong Liu, born in 1988, 185cm height, a Chinese female shot-putter, is the champion of the female shot put of China Throwing Game - Chengdu Site.

\section{B. Research Methods}

In the use of two Japanese High Speed Camera - JVC9800 (50 frame per second) to capture a moving object in simultaneous video. The two cameras were set separately at the right behind and right side of the throwing range, and the angle between the two cameras is $90^{\circ}$, and the Photo-frequency is $50 \mathrm{~Hz}$. Then using the 3D Signal TEC V1.0C video analysis software to deconstruct the movements of the athlete, with the body model of Matsui Shuji (21 articulation points and 16 segments). The original statistics was smoothly processed by the low - pass filter, and the cutoff frequency is $8 \mathrm{~Hz}$.

\section{RESEARCH RESULTS}

\section{A. Time Phasing}

Xiangrong Liu's shot-put movement is right-hand-throwing back gliding shot put. With a view of facilitating research, we break-down the movement into four moments, three stages as follow:

Four Moments:

- T1: the moment Liu's right foot off ground;

- T2: the moment Liu's right foot on ground;

- T3: the moment Liu's left foot on ground;

- T4: the moment the shot leave Liu's hand.

- Three Stages:

- T1-T2: Sliding Stage;

- T2-T3: Transitional Stage;

- T3-T4: Ending Movement Stage.

\section{B. Kinematics Analysis of Sliding Stage}

This stage starts from T1 to T2, through the movement of athlete's hip backward and left leg backswing actively. The main task of this stage is to obtain the key drivers towards the throwing direction, to achieve the level of low, smooth and fast Low, means the center of gravity of body should be low in the sliding stage; smooth, means to reduce the level of body's up and down to reach a smooth trace; fast, means the speed of wing and stretch and pedal should be fast. To ensure the presumed speed of shot and body's gravity is to lay a foundation of the optimum prior gesture to the second and the third stage.

There's a 0.16s's range between the VMAX of Liu's left knee and the moment of her right foot off ground. And the highest speed of her left leg wing comes before the moment of her right foot off ground; the speed comes nearly when her right leg pedals actively, which would have a positive effect on her right leg's speed and power.

In the sliding stage, the speed of shot should base on the speed of body's gravity. The highest speed of Liu's shot is $2.62 \mathrm{~m} / \mathrm{s}$, and the highest speed of her body's gravity is $1.90 \mathrm{~m} / \mathrm{s}$, and the difference of the two is $0.72 \mathrm{~m} / \mathrm{s}$. while the difference of that of Zhihong Huang and Xinmei Sui (two elite athletes of China) relatively is $0.06 \mathrm{~m} / \mathrm{s}$ and $0.10 \mathrm{~m} / \mathrm{s}$. Their sliding skills 
are excellent in this stage. Compared with them, Liu's difference in sliding stage is quite big, which indicates that the height of her body is quite high, and the skill of sliding is not ideal in this stage. And she needs more improvement.

\section{Kinematics Analysis of Transitional Stage}

This stage starts from T2 to T3, which are a continuation of the sliding stage and a foundation of the ending movement stage. It plays a transitional role in the whole course of shot put.

The primary mission of this stage is to continue the movement of athlete's body and the shot, to faster the transform from single support to double support and to convert the kinematic energy into elastic potential energy. Transitional skill is a crucial segment in the course of backward sliding shot put, which is regarded as the best gesture for drivers. And this kind of skill can help maintain even improve the initial speed for the ending movement stage. When right foot is on the ground, it's important to be inner $90^{\circ}$ for ankle joint, and in the meantime, the knee-joint and hip-joint should twirl rapidly. When they twirl rapidly, the upper part of body should not lift so quickly, and the non-swing arm should be relaxed and the left foot should insert quickly towards the stop board to reduce transitional time. With the series movements above, athlete's body comes into being a status of lasing gesture and a super-robot movement.

The speed of left hip should be faster than right hip when the right foot strikes the ground, which is the continuation of the sliding movement. A faster speed of left hip will profit the speed of left leg inserting the stop board. The speed of Xiangrong Liu's left hip is slower $0.17 \mathrm{~m} / \mathrm{s}$ than the speed of her right hip, which indicates Liu should speed up her left leg's inserting. When her left foot strikes on the ground, her right hip converts faster than her left hip about $0.31 \mathrm{~m} / \mathrm{s}$, which indicates that her right leg speeds up immediately when her left foot strikes ground. And this action will completely better her body to take on a gesture of lasing and a super-robot movement.

Transitional time can reflex the conversion effects between athlete's kinematics energy and elastic potential energy, which is an important kinematics parameter of transitional-skill evaluation. Liu costs $0.16 \mathrm{~s}$ in this stage, in the method of material reference, Chinese elite athlete Zhihong Huang $(21.52 \mathrm{~m})$ costs $0.106 \mathrm{~s}$ in her average. This comparison implies that Liu spends a quite long time for supporting and she needs to deduce her time in transitional period.

\section{Kinematics Analysis of Ending Movement Stage}

This stage starts from T3 to T4. In this stage, left swing leg should insert actively to brake, which is the base of energy transform. In the meantime, right leg should pedal, stretch and twist hip quickly to link all of the movement in a moment. The order of the power usage should start from up of the body to down, which means the non-throwing arm should stretch towards the top forward positively to ensure the correct direction and height of shot put and to promote the speed of shooting. The ending movement stage plays an important part in the throwing course and has a direct influence to athlete's final grades.

The angle out of hand of excellent female athlete is between $34^{\circ}$ to $38^{\circ}$ normally. Liu's angle out hand is $39.20^{\circ}$, which has a large difference from the excellent athletes, so her angle is not so reasonable. She should improve this in her daily exercise and to lower the angle to the reasonable range. Besides, the speed of Liu's out of hand in the last stage is $12.10 \mathrm{~m} / \mathrm{s}$ while that speed of the Chinese excellent athlete Lijiao Gong is $12.94 \mathrm{~m} / \mathrm{s}$, which states that Liu's speed is quite slow. The ending movement stage is the most important stage in shot-put skill, therefore, Liu should pay more attention on promoting the speed of her out of hand, which is also a difficulty that must be defeated in her way to a higher grade.

\section{CONCLUSIONS}

Sliding Stage: Compared with Chinese elite athletes, Liu's difference in sliding stage is quite big, which indicates that the height of her body is quite high, and the skill of sliding is not ideal in this stage. And she needs more improvement.

Transitional Stage: Compared with Chinese elite athletes, Liu's transitional time is quite long and the speed of her foot's insert is quite slow.

Ending Movement Stage: Compared with Chinese elite athletes, Liu's off-hand speed is quite slow and the angle is quite high.

\section{SUGGESTIONS}

Sliding Stage: in this stage, athlete's right leg should pedal actively to obtain the key drivers; to be slow, smooth and fast to reduce the difference between the speed of shot and the velocity of body gravity; control the height of body; refine sliding skills.

Transitional Stage: in this stage, athlete should enhance the exercising of legs' power; faster swing leg's inserting speed; deduce the supporting time of this stage.

Ending Movement Stage: in this stage, athlete should lower the angle of shot-off hand to control the angle within a reasonable area. In the meantime, faster the speeds of shot-off hand, and pay more attention on the order of the power between body and arms and legs, also for the position of the non-swing arms.

\section{REFERENCES}

[1] Guangbin Bai. The biomechanics Analysis of Back Sliding Shot Put Techniques of Ling Li[J]. Journal of Shandong Institute of Physical Education and Sports, 2008(3): 66-68.

[2] Jianchen Li, Jiabin Cui. Overall Pace of Shot Put Techniques of Zhihong Huang and Xinmei Sui[J]. Journal of Sports Sciences, 1993.(13)3: 82-85. 